\title{
Edema agudo hemorrágico de la infancia. Lesiones alarmantes de un cuadro benigno. Reporte de caso
} Acute hemorrhagic edema of infancy: alarming lesions of a benign condition.
Case report

\author{
Dr. Jorge Alvarado Socarras ${ }^{a}$ y Dra. Zhirly A. Fernandez Velosa ${ }^{b}$
}

\begin{abstract}
RESUMEN
Se reporta un caso de edema agudo hemorrágico de la infancia, en un lactante de 18 meses, después de un episodio de otitis media. El cuadro comenzó con máculas eritematosas en los muslos, seguidas de lesiones purpúricas en los brazos, las piernas y edema en los tobillos. Se interpretó, inicialmente, como urticaria, por lo que recibió esteroides. Sin embargo, las características clínicas fueron de edema agudohemorrágico dela infancia, una vasculitis leucocitoclástica benigna que se presenta en niños de entre 4 y 24 meses y que se caracteriza por fiebre, máculas y lesiones purpúricas. Estas se ubican, principalmente, en la cara, los lóbulos de las orejas y las extremidades, y se asocian, muchas veces, a edema. Los diagnósticos diferenciales son eritema multiforme, urticaria, vasculitis inducida por droga, enfermedad de Kawasaki, eccema infectado, meningococcemia y maltrato infantil, algunas de ellas, con riesgo de mortalidad. El manejo es conservador, sin embargo, los esteroides podrían ser una opción terapéutica.

Palabras clave: pediatría, vasculitis leucocitoclástica cutánea, esteroides.
\end{abstract}

\begin{abstract}
We report a case of acute hemorrhagic edema of infancy in an 18-month-old boy after an episode of otitis media. The clinical presentation begins with skin erythematous macules on the thighs, followed by purpuric lesions in arms, legs, and ankle edema. It was initially interpreted as urticaria, whereby steroids were indicated. However, the clinical feature was acute hemorrhagic edema of infancy, a benign leukocytoclastic vasculitis that occurs in children between 4 and 24 months of age and is characterized by fever, large purpuric palpable target-like skin lesions affecting the face, lobes of the ears, limbs and frequently associated with edema. Differential diagnosis includes erythema multiforme, hemorrhagic urticaria, druginduced vasculitis, Kawasaki disease, infected eczema, sepsis
\end{abstract}

a. Unidad Neonatal. Fundación Cardiovascular de Colombia. Grupo Organización Latinoamericana para Fomento de la Investigación en Salud (OLFIS).

b. Salud total EPS, Urbanización El Bosque.

Correspondencia:

Dr. Jorge Alvarado Socarras: Jorgealso2@yahoo.com

Financiamiento: Ninguno.

Conflicto de intereses: Ninguno que declarar.

Recibido: 2-2-2017

Aceptado: 19-6-2017 (either meningococcal or non-meningococcal) and child abuse. Some of them have risk of mortality. Management is conservative, however, steroids may be a therapeutic option. Key words: pediatrics, cutaneous leukocytoclastic vasculitis, steroids.

http: / / dx.doi.org/10.5546/aap.2017.e432

Cómo citar: Alvarado Socarras J, Fernandez Velosa Z. Edema agudo hemorrágico de la infancia. Lesiones alarmantes de un cuadro benigno. Reporte de caso. Arch Argent Pediatr 2017;115(6):e432-e435.

\section{INTRODUCCIÓN}

El edema agudo hemorrágico de la infancia (EAHI) es una vasculitis leucocitoclástica poco frecuente, posiblemente subdiagnosticada, a pesar de tener criterios diagnósticos bien definidos. ${ }^{1,2}$ Fue reportada hace más de 100 años por Snow en Estados Unidos (EE. UU.); sin embargo, recién en 1938, Finkelstein, en Europa, realizó la primera descripción detallada de la enfermedad. De ahí que se reconozca como síndrome de Finkelstein. ${ }^{2}$ Aunque muchos factores se relacionan con su etiología, aún no hay claridad sobre ella. A pesar de esto, lo que sí está definido es su comportamiento benigno y autolimitado en los niños que desarrollan el cuadro. Este se caracteriza por la tríada fiebre, edema de cara, pabellones auriculares y lesiones purpúricas como rosetas. Además, comparte características con la púrpura de Henoch-Schönlein (PH) y es este su principal diagnóstico diferencial (algunos autores la consideran una variante de esta enfermedad). A diferencia de la PH, en el EAHI, no hay, habitualmente, afectación visceral; ${ }^{1,3}$ pueden existir casos limítrofes entre ambas entidades. ${ }^{4}$ Típicamente, afecta a niños de entre 4 y 24 meses; sin embargo, hay reportes por debajo de esta edad, inclusive en el período neonatal, lo que hace el diagnóstico un poco más difícil..$^{3,5} \mathrm{Si}$ bien su curso es benigno y autolimitado, las lesiones pueden originar gran angustia a los padres e, incluso, al personal médico, ya que pueden simular enfermedades graves, como púrpura fulminans, meningococcemia, enfermedad de 
Kawasaki, fascitis necrotizante, erupción inducida por drogas, eritema multiforme y hasta maltrato infantil, entre otras. ${ }^{3} \mathrm{El}$ buen estado general de los pacientes es clave en el diagnóstico.

Se presenta a un lactante con un cuadro clínico compatible con EAHI. Se hizo un diagnóstico inicial de urticaria y se indicaron esteroides sistémicos. El paciente presentó buena evolución clínica, con rápida mejoría de las lesiones dermatológicas. Se realizó de forma retrospectiva un diagnóstico final de EAHI con una buena respuesta clínica al esteroide.

\section{Descripción del caso}

Lactante de 18 meses, previamente sano, sin antecedentes personales ni familiares relevantes, medicado por otitis media aguda, inicialmente, amoxicilina en dosis de $80 \mathrm{mg} / \mathrm{k}$ / día y luego amoxicilina-clavulanato en dosis similares, para un total de 17 días. A las 48 horas de terminado el esquema antibiótico, reapareció la fiebre, asociada a lesiones eritematosas en los muslos, por lo que consultó a Urgencias. Al momento de su ingreso, se encontraba febril $\left(38^{\circ} \mathrm{C}\right)$, con frecuencia cardíaca de $110 \mathrm{x}$ minuto, frecuencia respiratoria de 28 x minutos y saturación de oxígeno de 95\% en aire ambiental. En el examen clínico, se evidenciaron lesiones eritematosas, redondas, elevadas, con tendencia a ser confluentes, en la cara interna de los muslos. Otoscopia con signos aún de efusión en el oído medio derecho. En el Servicio de Urgencias, se hizo un diagnóstico de urticaria y se inició manejo con hidrocortisona de $5 \mathrm{mg} / \mathrm{k} /$ día cada 6 horas. Por las lesiones descritas y el cuadro febril, se decidió hospitalizar. Se realizó un hemograma: leucocitos de 14500/ $\mathrm{mm}^{3}$; N: 35\%; L: 60; E: $2 \%$; M: 3\%; plaquetas: $550000 / \mathrm{mm}^{3}$; proteína $\mathrm{C}$ reactiva (PCR):
$15 \mathrm{mg} / \mathrm{dl}$. En las primeras 24 horas, las lesiones se tornaron purpúricas, con predominio en los brazos y los tobillos, con posterior aparición de edemas (Figura 1). En su estancia, no hubo signos de respuesta inflamatoria, con signos vitales estables, con adecuada tolerancia de la vía oral, buena relación con el entorno y sin dolor ante la manipulación de extremidades ni ante la bipedestación. A las 48 horas, se observó una resolución significativa de las lesiones, y permanecieron solo algunas en los pies y edemas en resolución (Figura 2). Por los antecedentes y la evolución clínica, el cuadro se interpretó como EAHI y se dio el egreso. Se continuó con prednisona ambulatoria por 3 días. A las 48 horas posegreso, se verificó la resolución del cuadro.

Figura 2. A las 72 horas de evolución

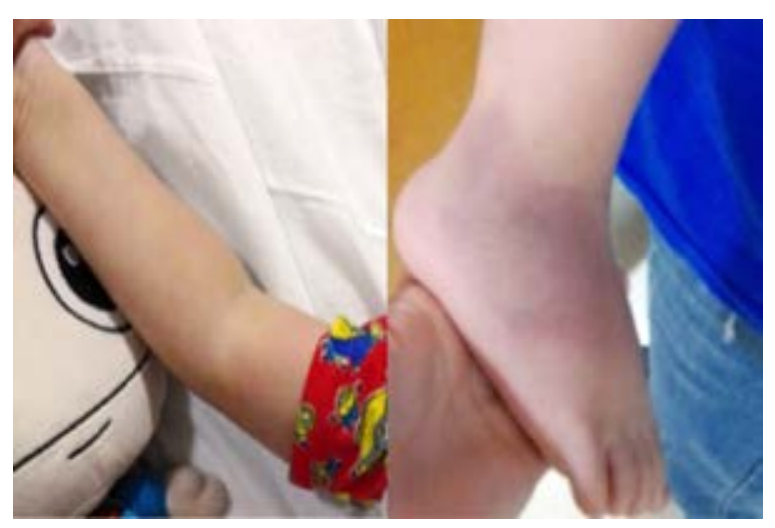

Posinicio de esteroides desde el día 1, se evidencia la resolución de lesiones equimóticas en los brazos y la mejoría del edema en las extremidades inferiores.

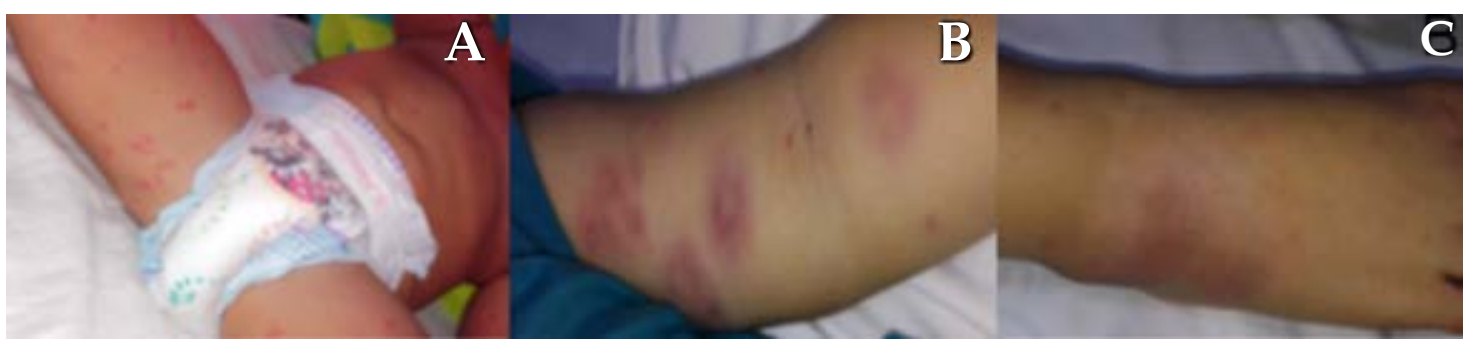

A. Día 1. Máculas eritematosas de menos de $1 \mathrm{~cm}$, múltiples, en la región proximal del muslo. B y C. Aparición 48 horas después de lesiones equimóticas con centro pálido en los brazos y lesiones similares en los pies, acompañados de edemas en los tobillos. 


\section{DISCUSIÓN}

El caso reportado corresponde a un caso típico de EAHI, con las características epidemiológicas, clínicas y evolutivas propias del cuadro. Su etiología es desconocida, pero se ha relacionado con cuadros infecciosos virales (citomegalovirus, coxsackie B4, rotavirus, herpes) o bacterianos previos, inmunizaciones y exposición a fármacos. Hoy se considera una vasculitis mediada por complejos inmunes. El antecedente de infección respiratoria (otitis media, rinofaringitis) refuerza el antecedente infeccioso en nuestro caso. Sin embargo, otras infecciones del tracto gastrointestinal e infecciones virales respiratorias domiciliarias se asociaron en otros casos reportados.,3

Si bien el diagnóstico es clínico, los estudios histopatológicos evidencian una extensa vasculitis con depósitos de fibrina, infiltrado perivascular de neutrófilos y eosinófilos, y se pueden observar depósitos de C3, inmunoglobulina A ( $\operatorname{Ig} \mathrm{A}$ ) en algunos pacientes. 3,6

$\mathrm{Al}$ no ser una condición sine qua non para el diagnóstico, se decidió no realizarlo en nuestro paciente, pero si hay dudas, puede ser de gran ayuda.

A los hallazgos clásicos pueden asociarse otras lesiones en la piel, como púrpura petequial, lesiones necróticas y urticariformes, y son menos frecuentes epistaxis y lágrimas de aspecto sanguinolento. ${ }^{7}$ Rara vez, aparecen artritis, sangrado gastrointestinal, eritema y edema escrotal. $^{8}$
Los estudios de laboratorio ayudan al diagnóstico diferencial, pero no hay hallazgos patognomónicos de la entidad. Puede encontrarse eosinofilia, trombocitosis y leucocitosis, elevación de reactantes de fases aguda, PCR, velocidad de sedimentación globular (VSG), hipocomplementemia (C4, C1q, CH50) y alteración transitoria de la pruebas de la función hepática. Las pruebas de coagulación son normales, pero puede existir elevación del fibrinógeno y dímero $\mathrm{D}$, al parecer, relacionado con afectación sistémica. ${ }^{2,9}$

Las lesiones de la piel pueden resultar alarmantes, por lo que deben realizarse los diagnósticos diferenciales pertinentes, basados, sobre todo, en los hallazgos clínicos y signos asociados que deben descartar otras entidades potencialmente graves (Tabla 1). Esto permitirá descartar enfermedades graves y dar tranquilidad a los padres sobre la condición y evolución final de esta enfermedad.

El manejo es sintomático y no existe terapia específica para este proceso dado su carácter benigno y autolimitado, cuya evolución no suele superar las 3 semanas. Sin embargo, en los últimos años, se planteó el uso de esteroides sistémicos como una alternativa para acortar el tiempo de las lesiones o en caso de compromiso sistémico, como orquitis y dolor articular. Inclusive, altas dosis de metilprednisolona ( $20 \mathrm{mg} / \mathrm{k} /$ día) fueron propuestas como una opción terapéutica, ${ }^{10}$ pero no puede ser una conducta de uso rutinario, ante la evolución benigna del cuadro.

TABLA 1. Signos clínicos de alarma ante la sospecha de edema agudo hemorrágico de la infancia

1. Púrpura y necrosis asociada a coagulación intravascular diseminada.

2. Fiebre alta, vómito, cefalea.

3. Edema acompañado de dolor, ampollas, decoloración, descamación.

4. Compromiso de mucosa oral, adenopatía cervical.

5. Inyección conjuntival.

6. Aparición de vesículas y/o ampollas.

7. Signos de respuesta inflamatoria.*

8. Evaluar compromiso visceral.

9. Irritabilidad, llanto continuo, rechazo al alimento y alteración neurológica.

* Presencia, al menos, de 2 de los siguientes 4 criterios (uno de ellos debe ser temperatura o recuento leucocitario anormal):

- Temperatura central $>38,5{ }^{\circ} \mathrm{Co}<36^{\circ} \mathrm{C}$.

- Taquicardia $>2$ desvíos estándar (DE) sobre el valor normal o bradicardia $\mathrm{p}<10$ (en ausencia de estímulos externos, medicamentos crónicos o estímulos dolorosos).

- Frecuencia respiratoria $>2 \mathrm{DE}$ sobre el valor normal o ventilación mecánica en un proceso agudo.

- Leucocitosis o leucopenia según la edad o $>10 \%$ de formas inmaduras.

* Respuesta inflamatoria sistémica. 
Nuestro paciente recibió esteroide por una interpretación diagnóstica inicial de urticaria, que luego se decidió continuar por la buena evolución clínica. Esto pudo haber acortado el tiempo del cuadro clínico y disminuyó en forma significativa la ansiedad de los padres. Pero, una vez que se tenga claridad en el diagnóstico, la conducta deber ser expectante y de manejo ambulatorio.

El principal objetivo de presentación de este caso es tener un conocimiento claro de esta patología, que genera gran angustia a los padres, y disminuir la realización de un número de intervenciones innecesarias, que incluyen la hospitalización.

El EAHI es una entidad benigna, que, en un primer momento, puede semejar cuadros potencialmente graves y puede existir subdiagnóstico de ella. Si bien el proceso es autolimitado, se han reportado recaídas. ${ }^{11}$ Llama la atención la ausencia de datos de seguimiento de casos, lo que dificulta asegurar la ausencia de complicaciones a largo plazo. Principalmente, en relación con posible compromiso renal, dado su gran similitud con PH. Tener presente su existencia, diagnóstico y evolución disminuirá angustias a los padres e, incluso, al personal de salud.

\section{REFERENCIAS}

1. Savino F, Lupica MM, Tarasco V, et al. Acute hemorrhagic edema of infancy: a troubling cutaneous presentation with a self-limiting course. Pediatr Dermatol 2013:30(6):e149-52.

2. Ceci M, Conrieri M, Raffaldi I, et al. Acute Hemorrhagic Edema of Infancy Still a Challenge for the Pediatrician. Pediatr Emer Care 2016 [Epub ahead of print].

3. Serra e Moura García C, Sokolova A, Torre ML, et al. Acute Hemorrhagic Edema of Infancy. Eur Ann Allergy Clin Immunol 2016;48(1):22-6.

4. Castro Piñeiro I, Hernández Santana J, Soler Cruz E, et al. Edema agudo hemorrágico del lactante. Actas Dermosifiliogr 1998;89(4):189-93.

5. Checa Rodríguez R, Carabaño Aguado I, Álvarez Fernández B. Edema agudo hemorrágico en un neonato. Rev Pediatr Aten Primaria 2015;17(66):151-3.

6. Oliveira JA, Lopes L, Fraga A, et al. Acute Hemorrhagic Edema of Infancy: A Rare Cause of Purpuric Exanthema. J Pediatr 2015;166(2):498.e1.

7. Mreish S, Al-Tatari H. Hemorrhagic Lacrimation and Epistaxis in Acute Hemorrhagic Edema of Infancy. Case Rep Pediatr 2016;2016:9762185.

8. Cunha DF, Darcie AL, Benevides GN, et al. Acute Hemorrhagic Edema of Infancy: an unusual diagnosis for the general pediatrician. Autops Case Rep 2015;5(3):37-41.

9. Alhammadi AH, Adel A, Hendaus MA. Acutehemorrhagic edema of infancy: a worrisome presentation, but benign course. Clin Cosmet Investig Dermatol 2013;6:197-9.

10. Risikesan J, Koppelhus U, Steiniche T, et al. Methylprednisolone Therapy in Acute Hemorrhagic Edema of Infancy. Case Rep Dermatol Med 2014;2014:853038.

11. Roy KP, Madke B, Kar S, et al. Acute Hemorrhagic Edema of Infancy. Indian J Dermatol 2015;60(6):624-5. 